Paper No. 1-2

\title{
THE PHYSICAL THEORY OF NOISE IN REACTORS AND REACTOR-LIKE SYSTEMS
}

\author{
R. K. OsBoRN and S. YIP* \\ University of Michigan, Ann Arbor, Michigan
}

THE investigations to be reported here are primarily concerned with the theoretical basis for the phenomenological interpretation of measurements of fluctuations and correlations in neutron distributions. Thus a logical (rather than phenomenological) derivation of kinetic equations purportedly descriptive of these phenomena is developed from first principles.

The derivation proceeds deductively, albeit via a route strewn with approximations -most of which are qualitatively interpretable, but few of which have been quantitatively estimated-from the quantum Liouville equation. Operators whose expected values are manifestly interpretable as singlet and multiplet densities and cross densities for the various particle distributions requiring description are constructed along lines suggested by ONo (1954) in his studies of the kinetic theory of neutral gases, and employed elsewhere in a derivation of kinetic equations for plasmas (OsBORN, 1963). The deduction of equations to describe these densities requires some-but fortunately only some-knowledge of the Hamiltonian for the system, which, of course, is not at present fully understood. The equations ultimately obtained are complete in the sense that cross sections, scattering and fission frequencies, etc. emerge naturally with analytical representations which do not seem to differ significantly from those conventionally assumed. In particular, the equation for the neutron singlet density is, after a few unimportant approximations, identical to the one usually employed in reactor analysis.

By successive approximation these equations are then brought into contact with those presented and studied by MatTHES (1962) and Pluta (1962). In this process two points of possible significance are noted. First, useful formulas for the spacedependence of doublet densities in systems characterized by spatially uniform singlet densities are obtained. $\dagger$ Second, and perhaps more important, it is demonstrated that the cross sections appearing in the equations for the doublet densities and cross densities, when reduced to the working level by means of the usual 'point-reactor' approximations (PLUTA) differ from those appearing in the corresponding equations for the singlet densities. Thus the effort to deduce kinetic parameters for reactors from measurements on steady state systems must proceed with caution.

\section{REFERENCES}

MATTHES W. (1962) Nukleonik 4, (5) 213.

ONo S. (1954) Prog. Theor. Phys. (Kyoto) 12, 113.

OSBORN R. K. (1963) Phys. Rev. 130, 2142.

PluTA P. (1962) Unpublished thesis, University of Michigan.

* Now with Cornell University, Ithaca, New York.

$\dagger$ The existence of such space-dependence was pointed out to the authors by Prof. F. SHuRE. 